International Journal of Bifurcation and Chaos, Vol. 10, No. 5 (2000) 1115-1125

(c) World Scientific Publishing Company

\title{
SELF-ORGANIZATION IN NONRECURRENT COMPLEX SYSTEMS
}

\author{
PAOLO ARENA, RICCARDO CAPONETTO, \\ LUIGI FORTUNA* and ALESSANDRO RIZZO \\ Università di Catania, Dipartimento Elettrico Elettronico e Sistemistico, \\ Viale A. Doria 6, 95125 Catania, Italy \\ MANUELA LA ROSA \\ ST Microelectronics - Soft Computing Group - Catania site, \\ Stradale Primosole 50, 95100 Catania, Italy
}

Received August 18, 1999; Revised September 20, 1999

\begin{abstract}
In this paper, systems formed by networks of simple nonlinear cells are studied. Using lattice models, some of the fundamental features of complex systems such as self-organization and pattern formation are illustrated. In the first part of this work, a lattice of identical Chua's circuit is used to experimentally study its global spatiotemporal dynamics, according to the variation of some macroparameters, like the coupling coefficient or the neighboring dimension. The second part of the paper deals with the remarkable improvements regarding regularization and pattern formation, obtained in networks of nonlinear systems by introducing some spatial diversity, especially generated by deterministic, unpredictable dynamics. Simulation results show that synchronization and self-organization occur in networks with a few nonlocally connected cells, with irregular topology and small spatial diversity.
\end{abstract}

\section{Introduction}

Vast lattices of simple nonlinear units have recently become an important topic in modeling actual complex phenomena in several fields like astronomy, sociology, epidemiology or information processing [Gell-Mann, 1994]. Complex systems are often seen as networks of simple nonlinear cells of the same kind, connected according to several topologies. Although constituted by a great amount of small units, they denote a global behavior that can usually be described via a small number of macroscopic parameters [Kaneko, 1990]. In this work, the self-organization phenomena which take place in a network of locally interconnected chaotic systems are illustrated. The problem is not new in literature [Braiman et al., 1995]: these phenomena have often been emphasized in locally connected systems with a remarkable number of cells, generally improving the self-organization and pattern formation capability. The aim of this communication is to investigate how the global dynamics is influenced by the variation of important global parameters, like the coupling strength among cells and the connection topologies, including also the neighboring dimension, i.e. the number of cells directly connected to a given cell. The first obtained result concerns the drastic decrease of the number of cells required for self-organization when nonlocal connections are realized. Moreover, the effects of the introduction of slight spatial diversity throughout the cells are shown in the second part of this work. It is also shown that, using a smaller

*E-mail: lfortuna@dees.unict.it 
number of cells than the previous case, selforganization and pattern formation capabilities are improved if a small dissymmetry is introduced among the cell parameters. Remarkable improvements concerning self-organization and pattern formation are further achieved if the dissymmetry is generated by a deterministic, unpredictable source, like the sampling of a chaotic attractor.

\section{Analysis of Chua's Circuit Based Networks}

The basic cell adopted to carry out experiments through this work is the well-known Chua's circuit [Madan, 1993]. In this section, the behavior of a complex system constituted by a network of identical Chua's circuits is characterized according to the variation of some global parameters. The fundamental cell of the structure is described by the following adimensional equations:

$$
\begin{aligned}
\dot{x} & =-x+y+z \\
\dot{y} & =-\beta x \\
\dot{z} & =\alpha(x-h(z)) \\
h(z) & =a z-k z^{3}
\end{aligned}
$$

For a certain range of the parameters $\alpha, \beta, a$, and $k$, the system (1) exhibits a chaotic behavior, with a double scroll attractor in the phase space. In particular, for the experiments carried out in this paper, parameters have been set to $\alpha=6, \beta=8$, $a=-1.1429$, and $k=0.0635$. Cells of the form (1)

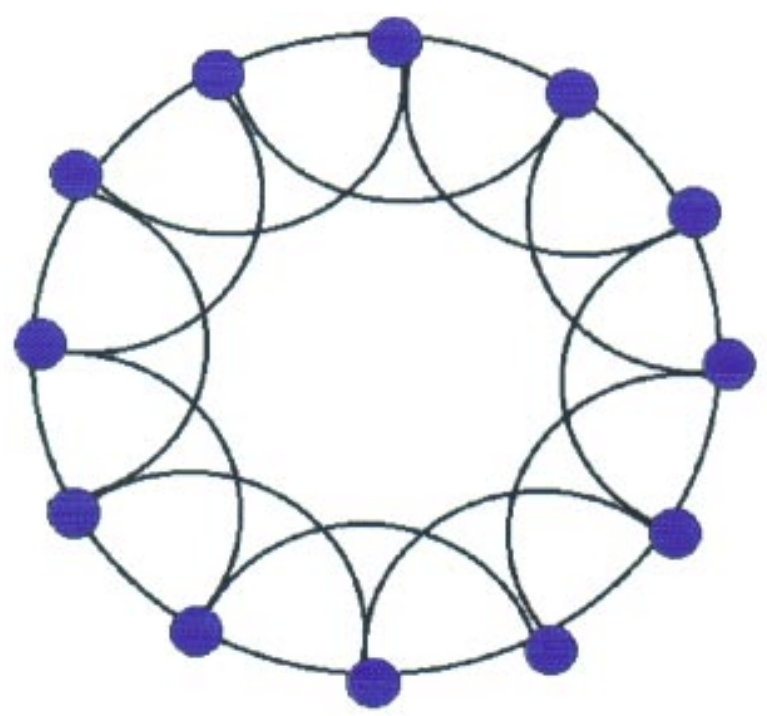

Fig. 1. Structure of the macrosystem with $C=4$. can be connected in a particular topology, basically constituted by a ring where additional connections to some further cells are introduced. The number of cells directly connected to a given cell is called neighboring size, and indicated with $C$ in the following. A typical topology, for $C=4$, is shown in Fig. 1.

Couplings are realized with linear diffusion-like terms, for which the strength is expressed by the variable $D$. It is important to notice that, as Chua's system can be easily implemented with an electronic circuit [Manganaro et al., 1999], a coupling term can be easily realized by using a simple linear resistor. Calling $B(i)$ the set of $C$ neighbors of the generic $i$ th cell, the dynamics of a coupled cell can be expressed by the following equation:

$$
\begin{aligned}
\dot{x}_{i} & =-x_{i}+y_{i}+z_{i}+D\left(\sum_{j \in B(i)} z_{j}-C x_{i}\right) \\
\dot{y}_{i} & =-\beta x_{i} \\
\dot{z}_{i} & =\alpha\left(x_{i}-h\left(z_{i}\right)+D\left(\sum_{j \in B(i)} x_{j}-C z_{i}\right)\right) \\
h\left(z_{i}\right) & =a z_{i}-k z_{i}^{3} \quad i=1 \ldots N
\end{aligned}
$$

Thus, the other important macroparameters that are useful to globally describe the behavior of the network as a whole are the coupling strength $D$ and the total number of cells $N$.

The first part of our study deals with the analysis of the dynamical behavior of a network of identical circuits versus the neighborhood size $C$. To our purposes, a network of 50 Chua's circuits, with fixed diffusion coefficient $D=0.01$, starting from random initial conditions, has been adopted. Major results are presented by colored maps, coding the evolution of the $x_{i}$ state variables versus time. For small values of $C(C=2$, corresponding to the plain ring configuration, and $C=6$ ), the network exhibits disorder both in time and space, as it is shown in Figs. 2 and 3. Increasing the $C$ parameter to $C=12$, some order and an initial pattern formation take place, as it is shown in Fig. 4. Finally, Figs. 5 and 6 show that, for $C=14$ or more, a perfectly synchronized behavior arises in which all the cells oscillate at the same frequency.

The behavior of the network versus the variation of the total number of cells $N$ is now investigated. It has been experimentally noticed that the network dynamics is insensitive to the 


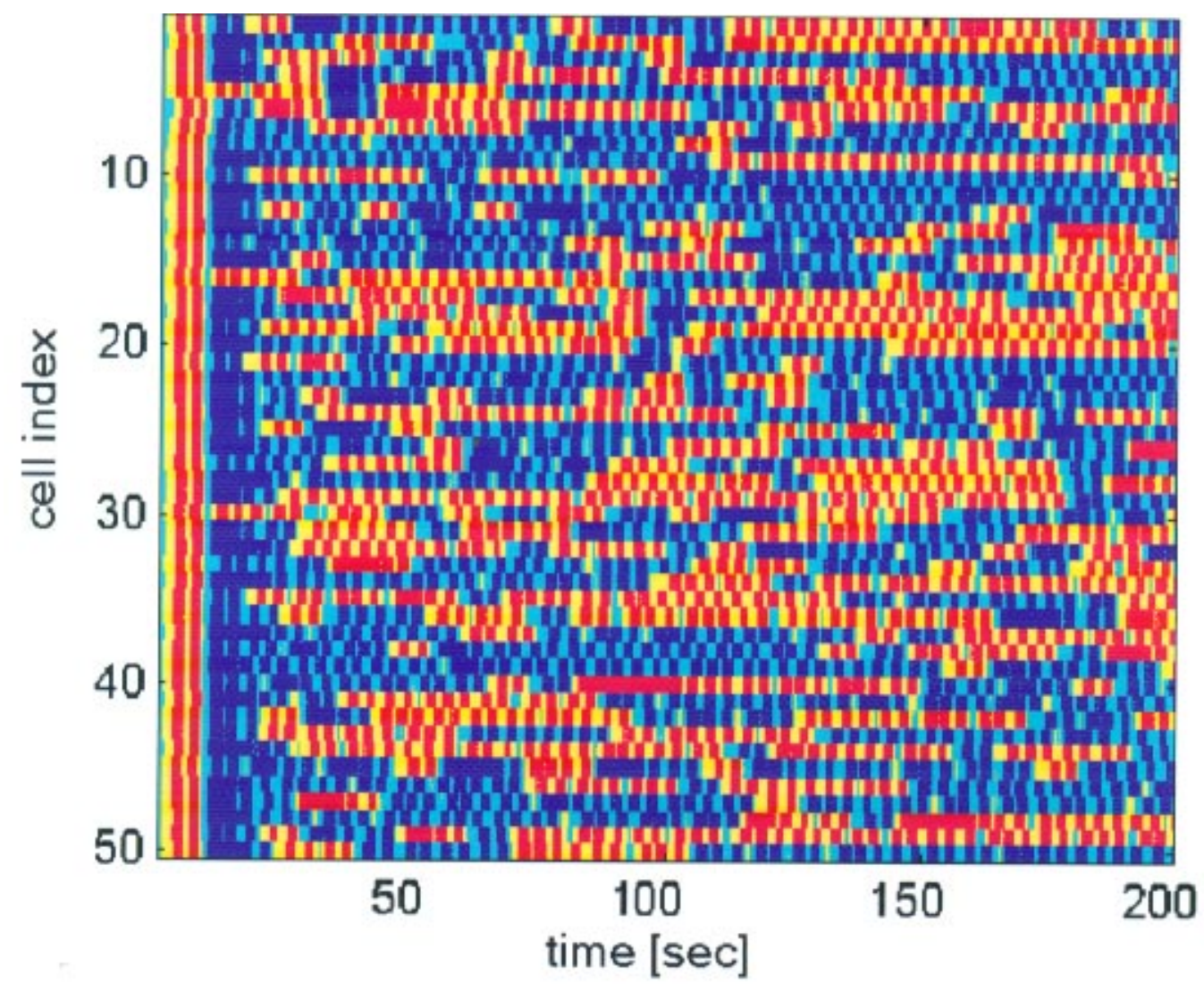

Fig. 2. Disorder with array configuration $(C=2)$.

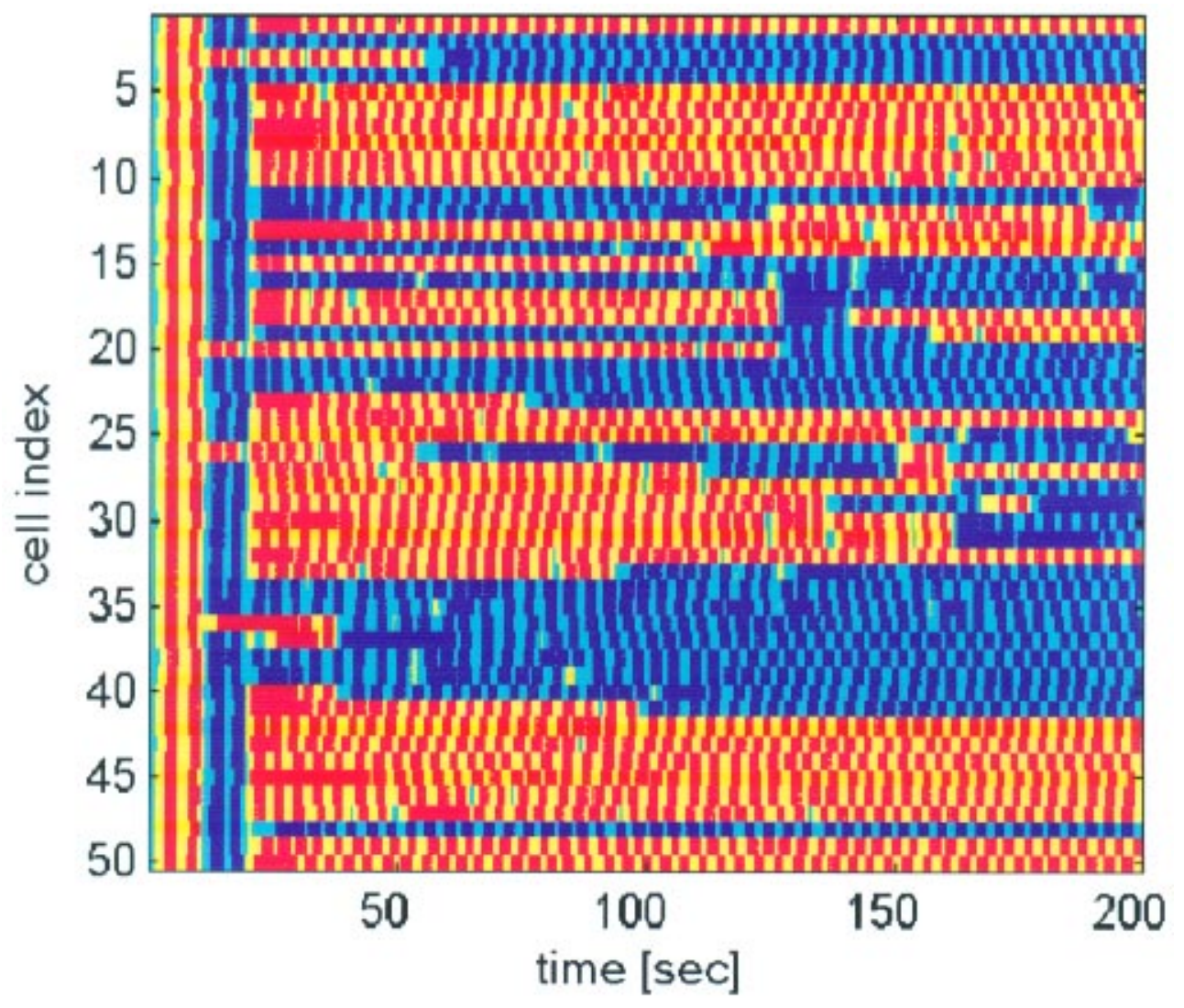

Fig. 3. Spatiotemporal chaos with neighborhood size $C=6$. 


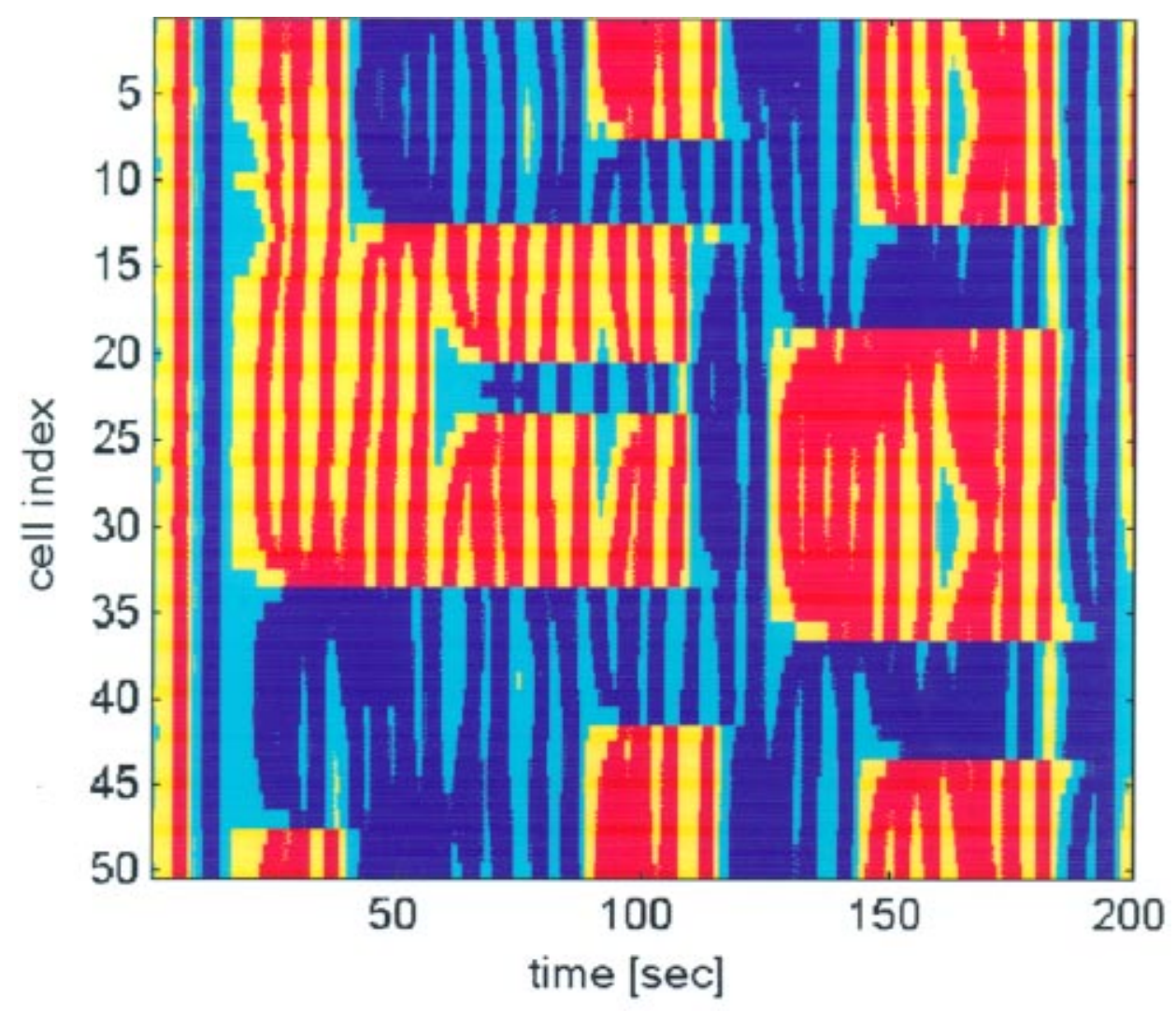

Fig. 4. Pattern formation with neighborhood size $C=12$.

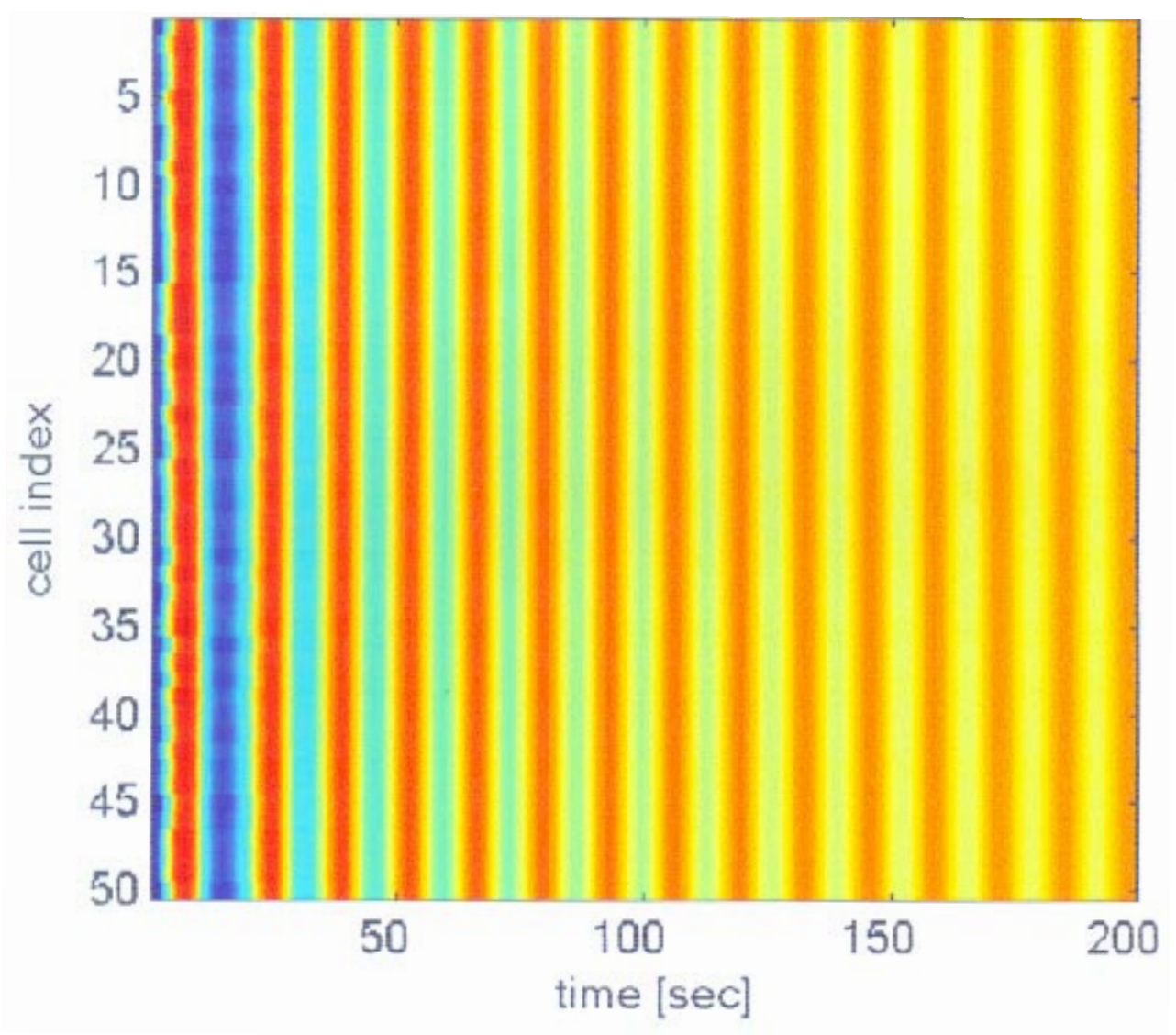

Fig. 5. Ordered structures arising with neighborhood size $C=14$. 


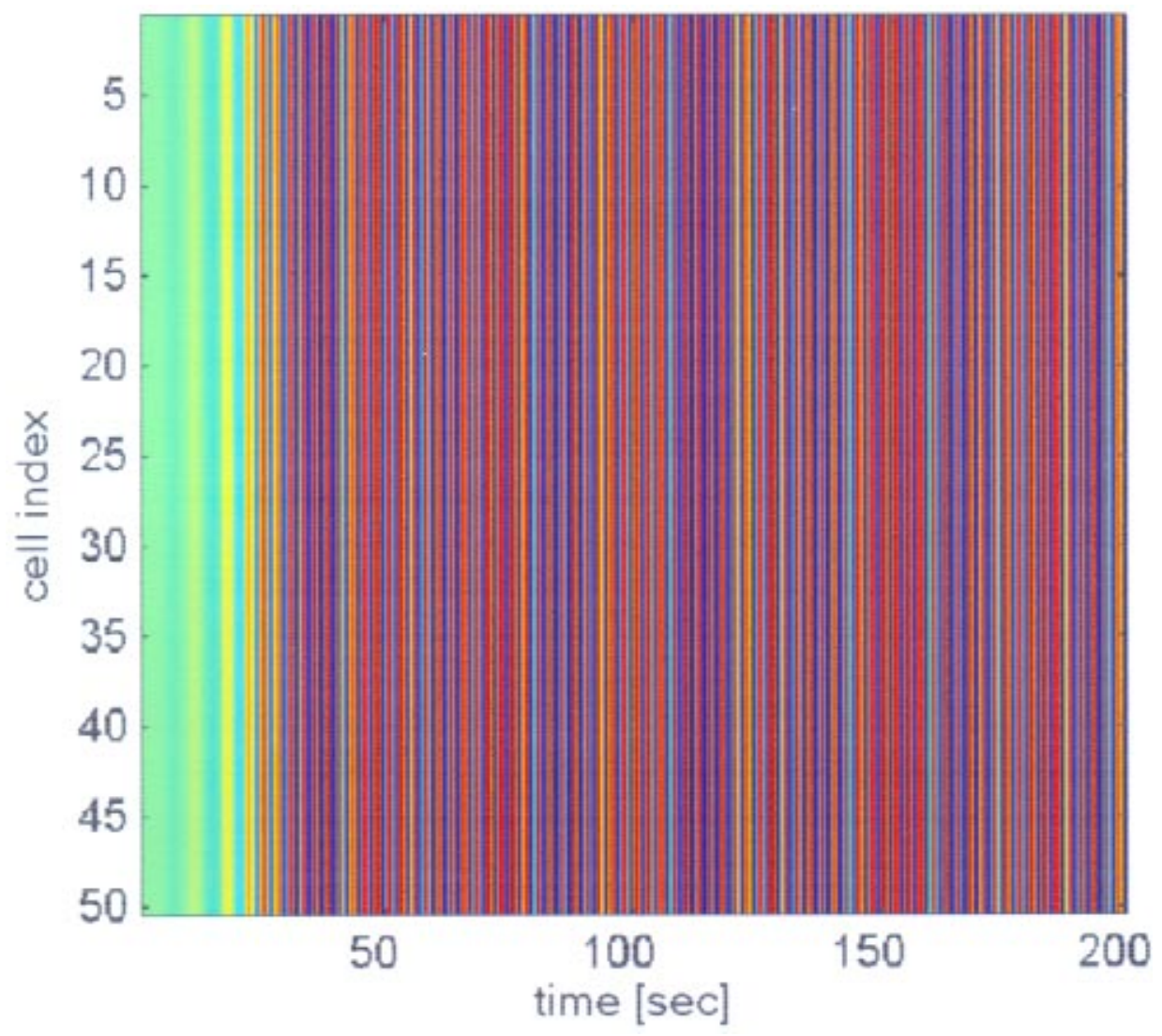

Fig. 6. Synchronized behavior with neighborhood size $C=16$.

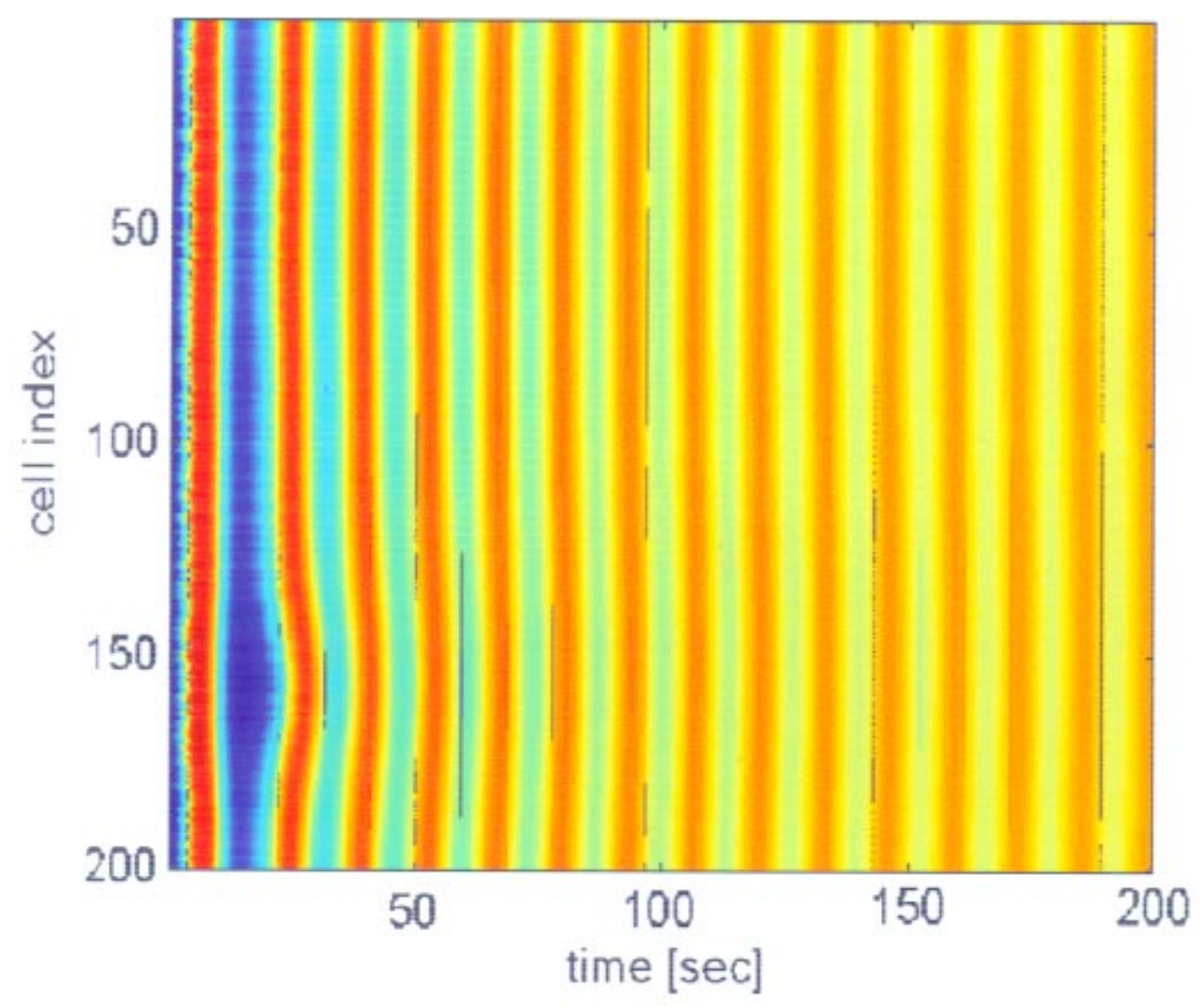

Fig. 7. Ordered behavior of 200 cells with neighborhood size $C=14$. 


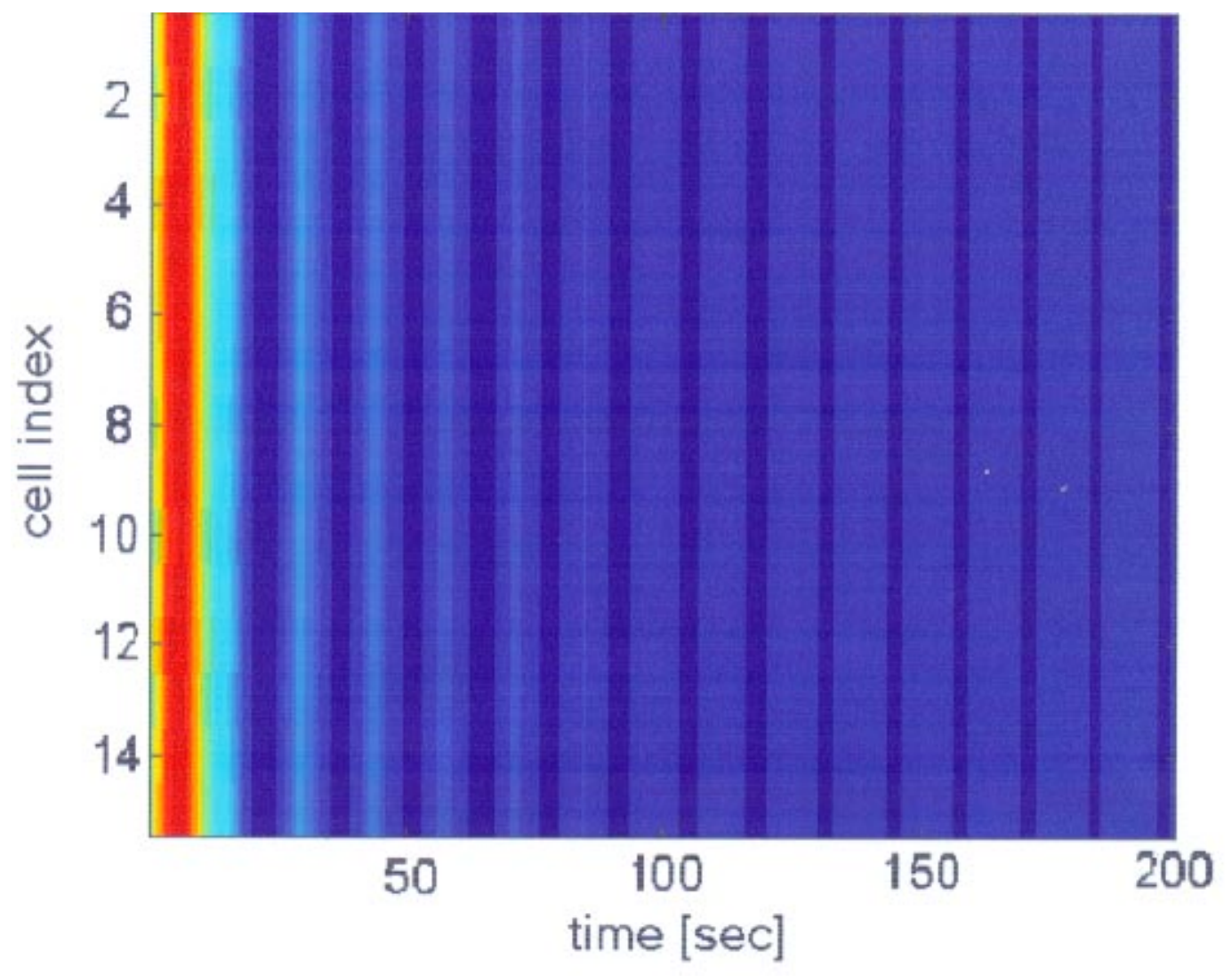

Fig. 8. Synchronized behavior for $C=N-1=14$.

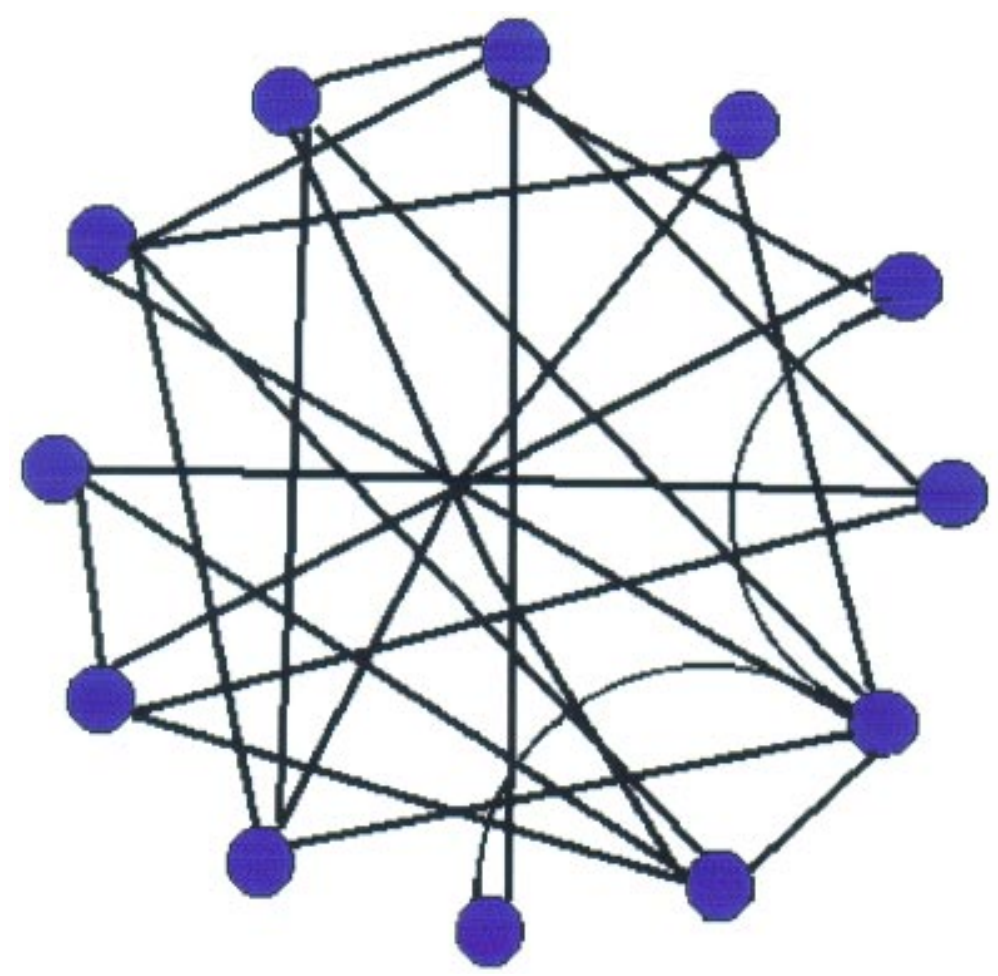

Fig. 9. Network irregular configuration. 


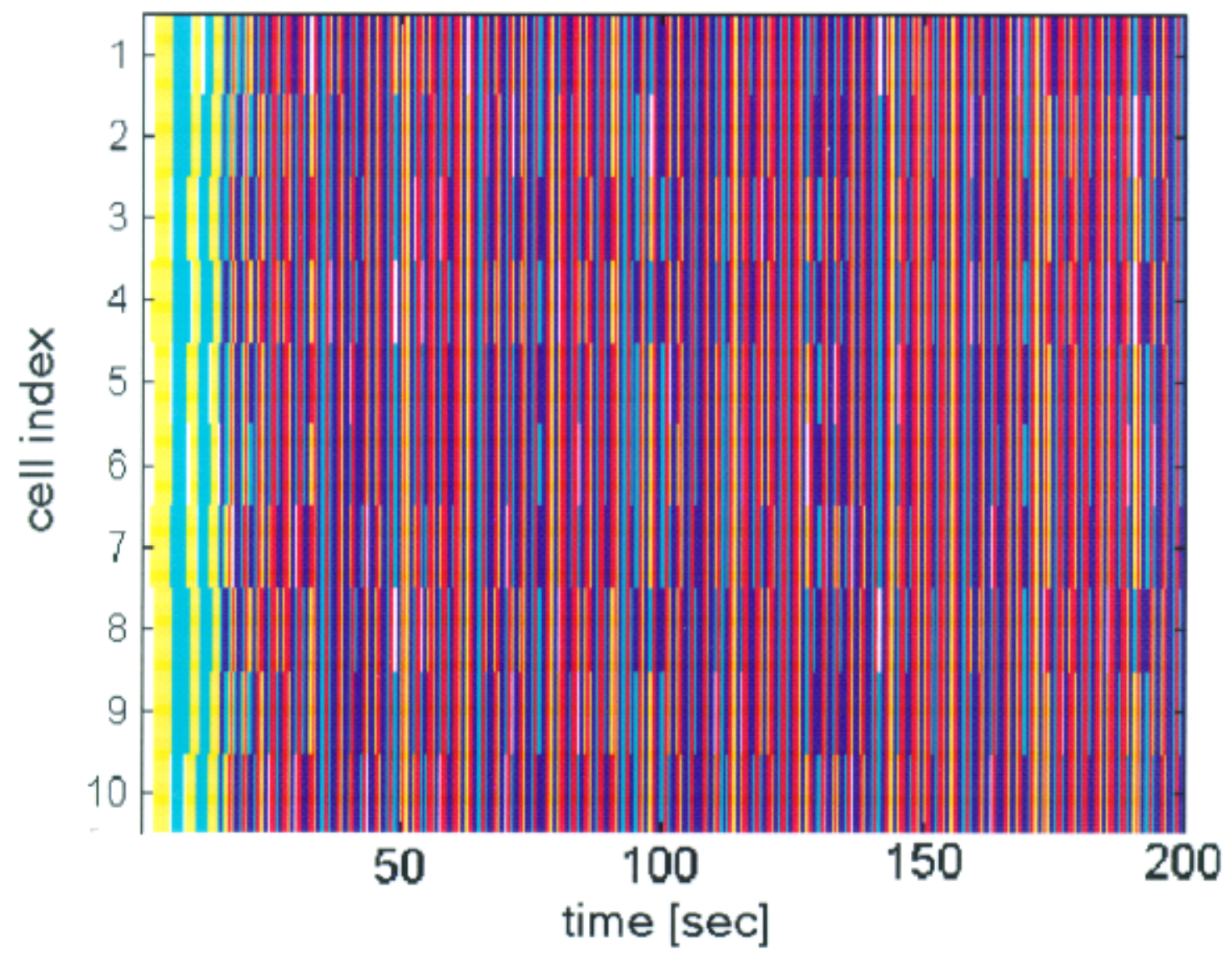

Fig. 10. Synchronization with irregular configuration for $C=9$.

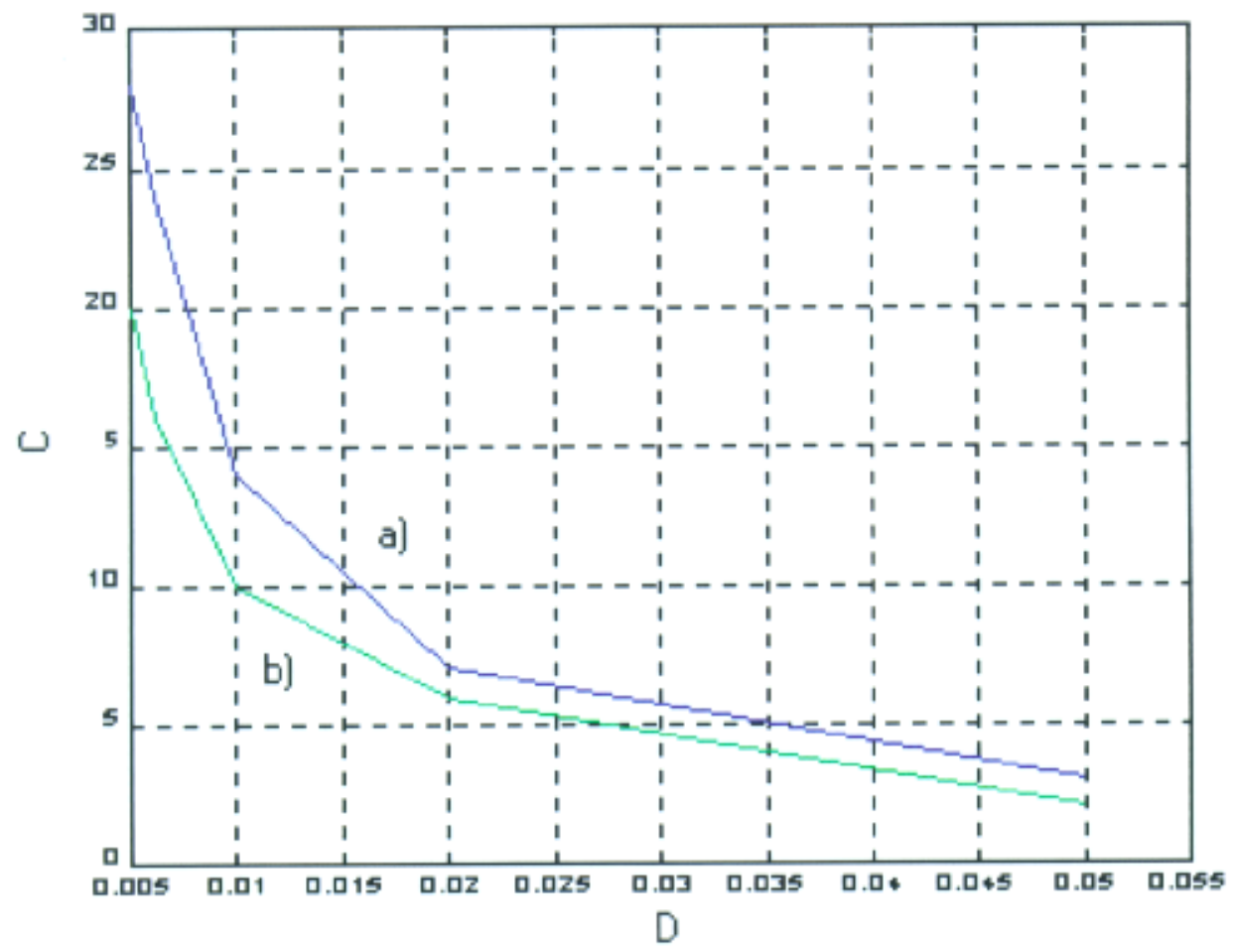

Fig. 11. Neighborhood size versus coupling strength with identical cells. 
variation of $N$. To emphasize this phenomenon, Fig. 7 shows the behavior of a network with $N=200$ cells, connected with a neighboring size $C=14$. It is clear that this dynamics is identical to that shown in Fig. 5, where $N=50$ cells have been connected with a neighboring size $C=14$. A remarkable result, depicted in Fig. 8, is that selforganization already occurs for a number of cells $N=15$, that is the minimum number of cells needed to obtain $C=N-1=14$, with a regular connection scheme like that reported in Fig. 1.

Self-organization is typically achieved in these kinds of networks when sufficient information is exchanged among cells. This has been obtained so far by increasing the neighboring size $C$. As the connection strength $D$ regulates the information exchange among cells, this parameter also seems to be crucial with respect to self-organization capabilities. Intuitively, if the connection strength $D$ increases, the minimum neighboring size $C$ to achieve self-organization and pattern formation has to decrease, because more information is supplied via the existing connections. Moreover, the introduction of disorder in the network configuration [Watts \&
Strogatz, 1998], as shown in Fig. 9, lead us to discover self-organization with fewer cells. In particular, self-organization phenomena occur for $C=$ $N-1=9$, as shown in Fig. 10 .

Figure 11 illustrates the trend of the minimum neighboring size $C$ versus the connection strength $D$, when global self-synchronization is achieved. Curve (a) refers to the regular topology, whereas curve (b) characterizes the irregular one, obtained by an irregular rewiring of the network. It can be clearly noticed that a good improvement in terms of self-synchronization capability is always achieved by adopting irregular topologies.

\section{Study of Dissymmetry Effects in Complex System Dynamics}

This section deals with Chua's circuit based networks in which some spatial dissymmetry is introduced. Nonrecurrence is one of the main features of real complex systems. All cells constituting a whole are actually slightly different in some parameters. Under this perspective, diversity plays a funda-

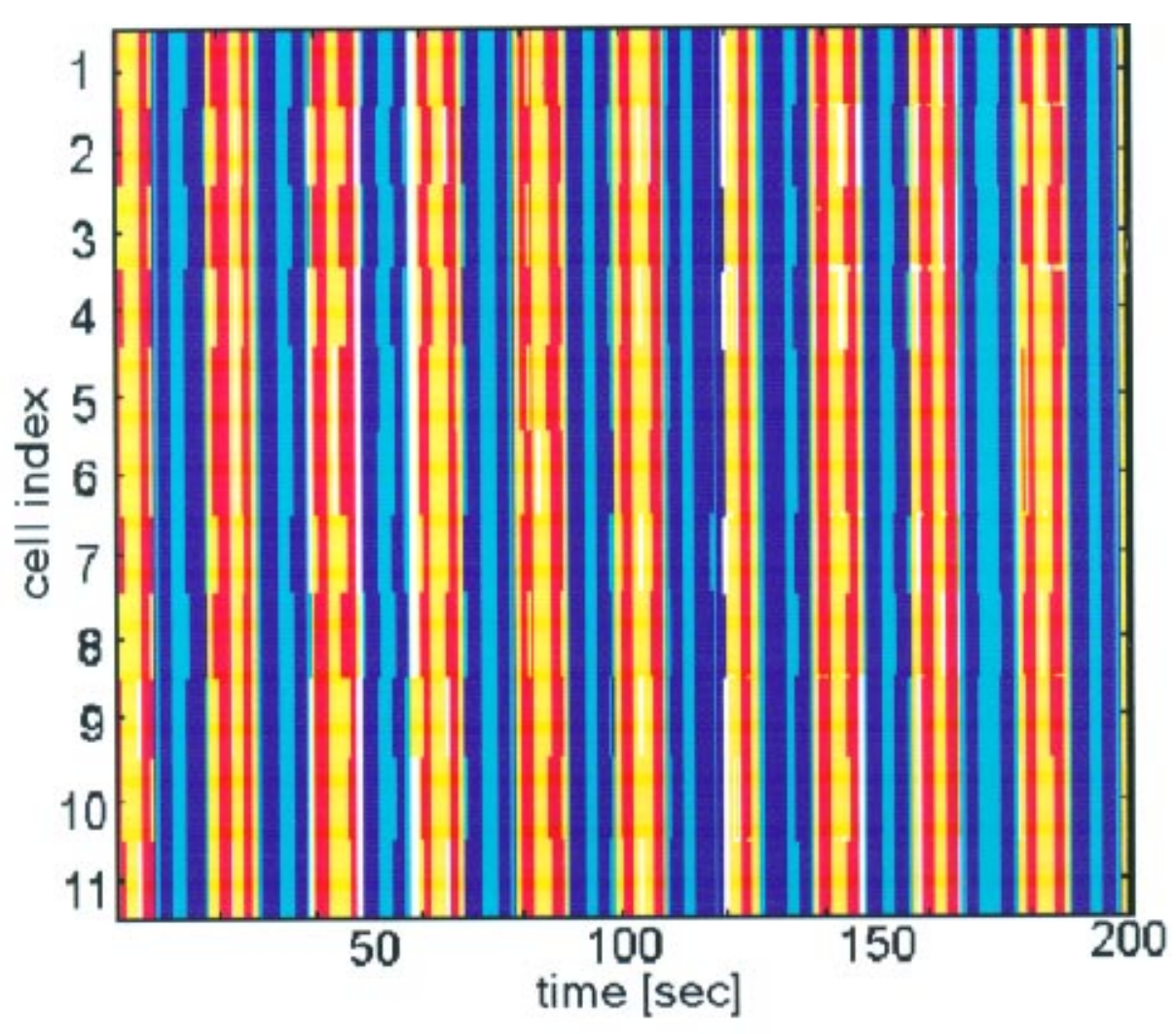

Fig. 12. Self-synchronized circuits with random disorder and $C=10$. 


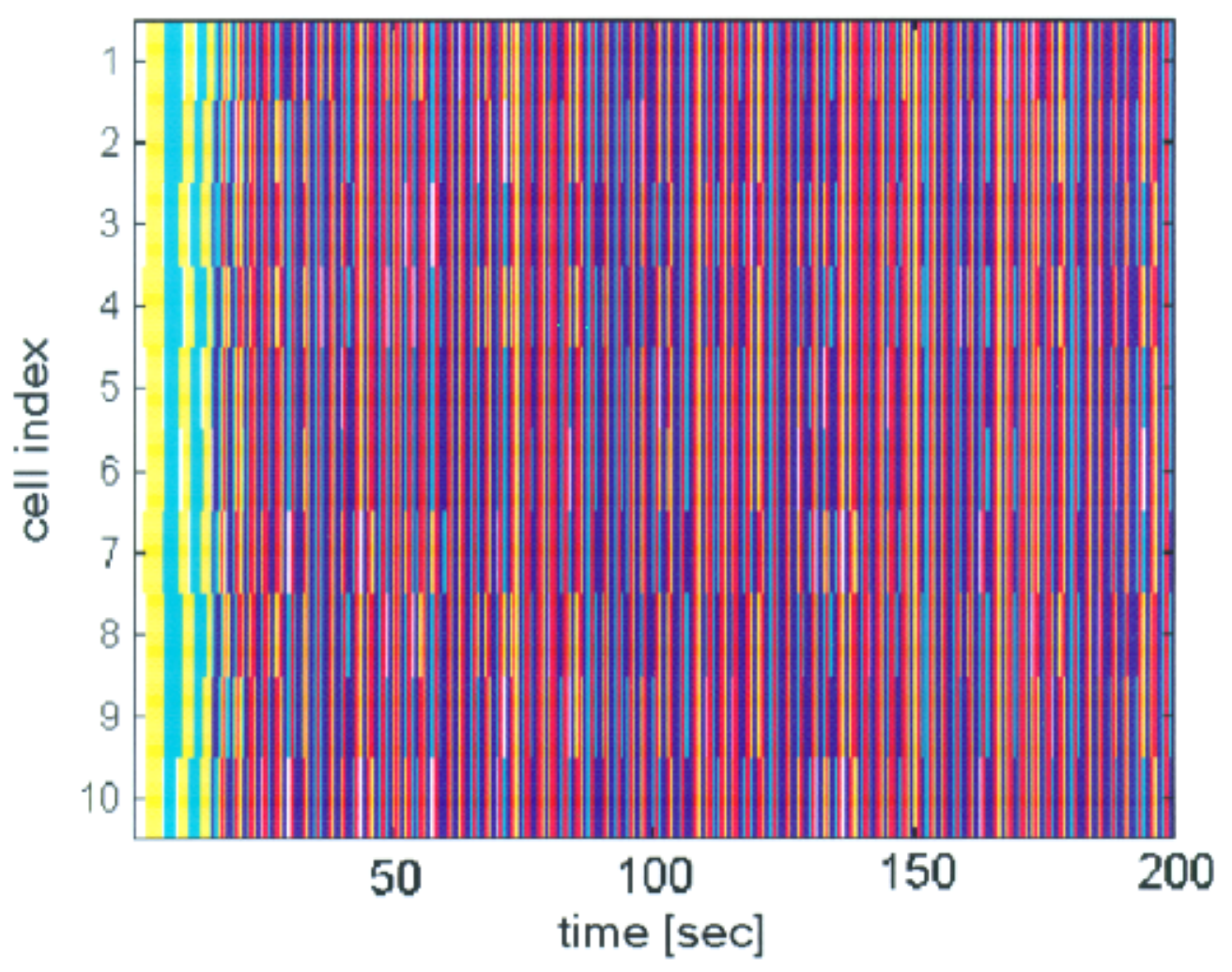

Fig. 13. Self-synchronized circuits with chaotic disorder and $C=9$.

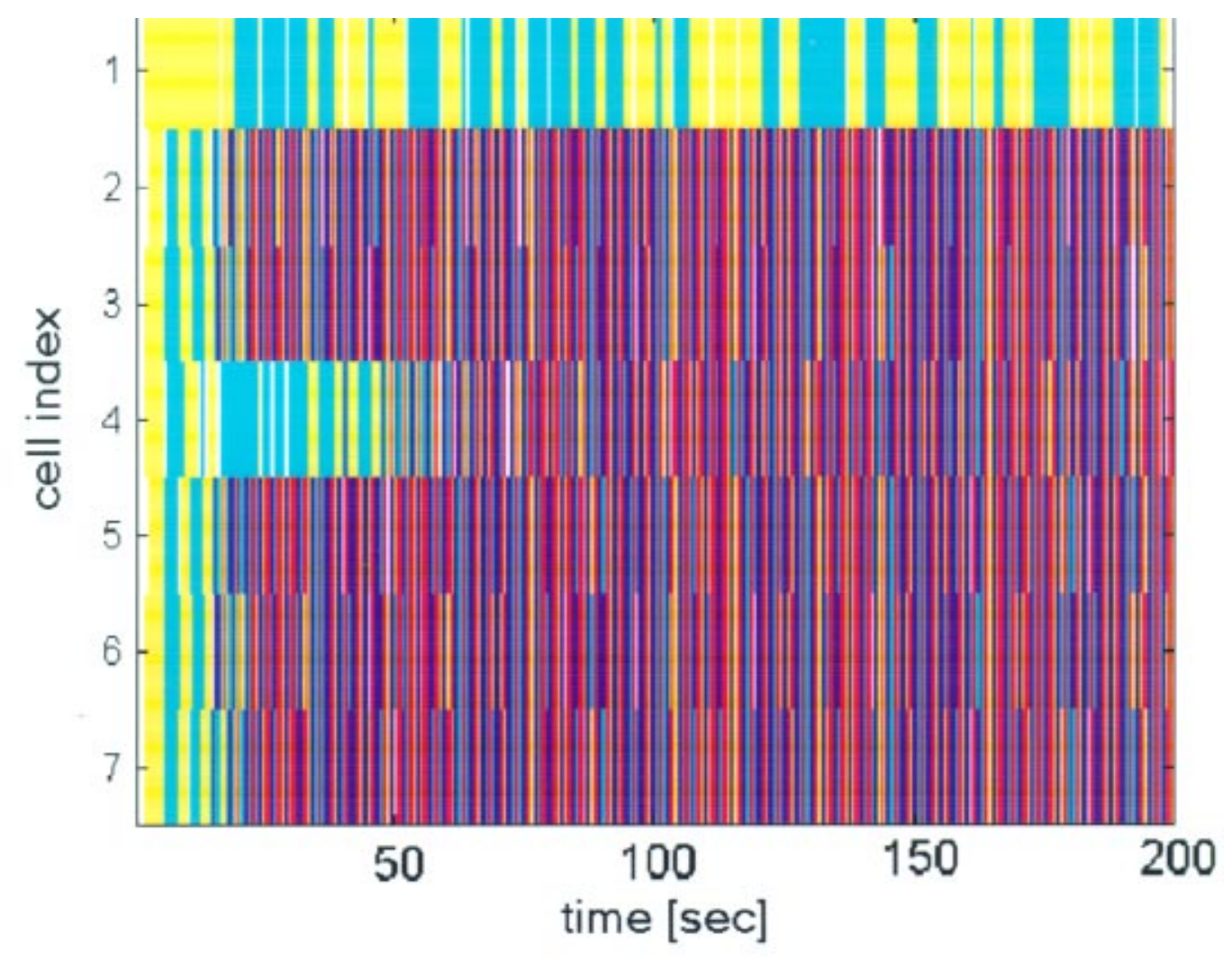

Fig. 14. Self-organized circuits with chaotic disorder and irregular topology for $C=6$. 


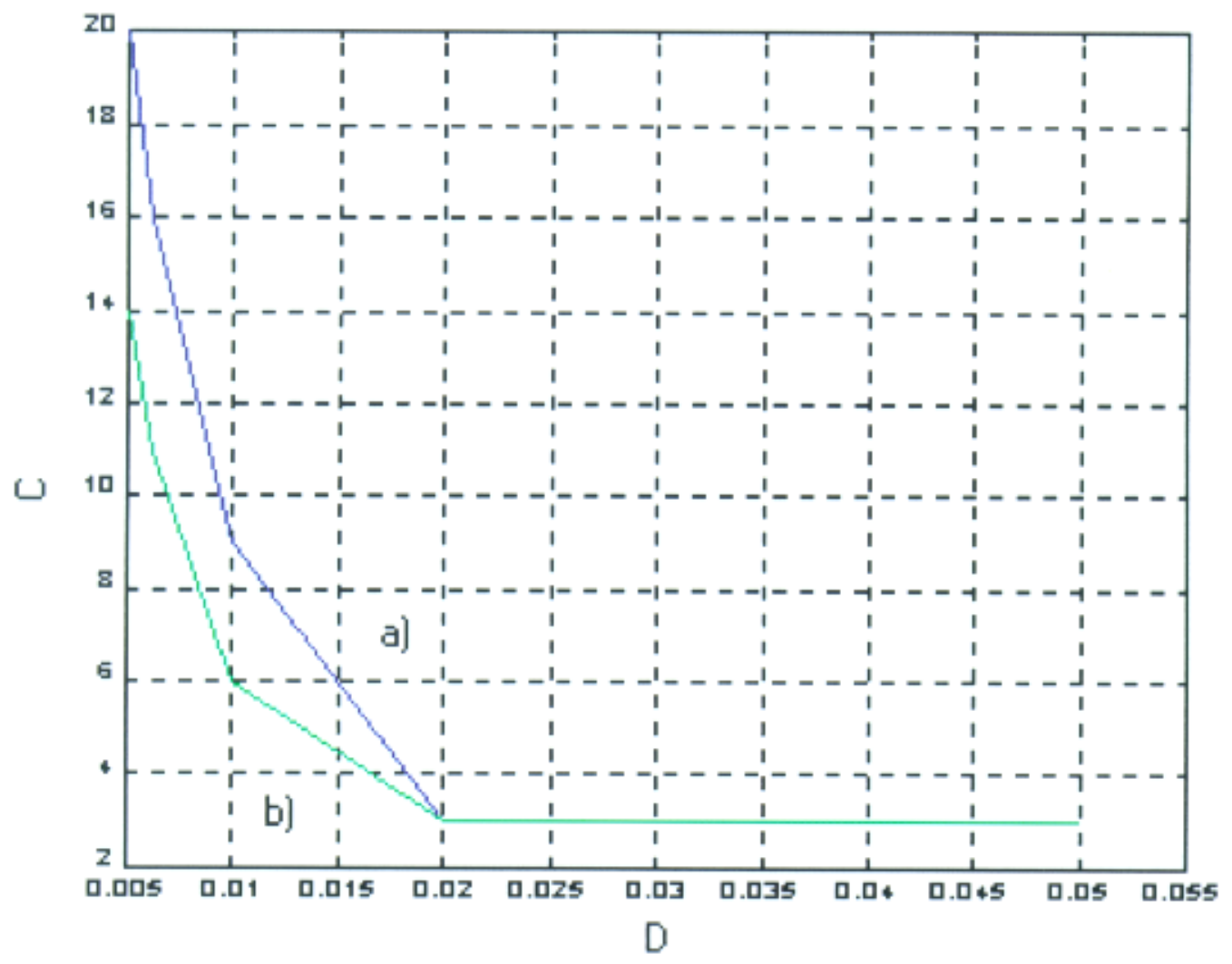

Fig. 15. Neighborhood size versus coupling strength after introducing the spatial diversity.

mental role in evolution and adaptation in nature. Some interesting work in the field of nonrecurrent systems has been recently carried out by generating diversity through classical stochastic processes [Braiman et al., 1995], but an intriguing hypothesis is now taking place: diversity generated by deterministic, although unpredictable dynamics, dramatically improves the aptitude to self-organization and pattern formation [Arena et al., 1999]. In this section, we emphasize this result by adopting as a reference model the one described by Eq. (2).

In the previous section, it has been shown that network (2), with a connection coefficient $D=0.01$, needs a neighboring size of $C=14$ and a minimum number $N=15$ to exhibit self-synchronization, as depicted in Fig. 5. As insensitivity with respect to the total number of units $N$ has been experimented, let us focus the attention on massively connected structures (i.e. all-to-all connections), fully representative of every network in which $N \geq C$.

The first experiment that has been carried out is perturbing the $\alpha$ parameter of system (2) by introducing a small spatial dissymmetry around $10 \%$ of the nominal value $\alpha=6$. The dissymmetry has been generated by a classical random generator with uniform distribution. Introducing this kind of disorder, the minimum neighboring size to obtain selforganization decreases to $C=10$, as it is shown in Fig. 12.

A further improvement in the self-organization phenomenon is obtained if diversity is generated by deterministic rules. To generate the diversity, a logistic function chaotic attractor has been sampled, and the obtained values have been scaled in order to impose a variation around $10 \%$ of the nominal value $\alpha=6$. This strategy leads to a minimum neighboring size of $C=9$. The dynamics of this self-organized system is shown in Fig. 13.

Further, the previous study has been reproposed by introducing also in this network the irregular topology. It appears that in this case the minimum neighboring size becomes $C=6$, and consequently $N=7$ (Fig. 14). The minimum neighboring size versus connection strength, when dissymmetry is applied, is shown in Fig. 15, where curve (a) represents the results related to networks with regular topologies, and (b) refers to irregular rewired networks. 


\section{Conclusions}

In this work, complex systems have been analyzed from a global point of view, focusing particularly onto their self-organization and pattern formation capabilities. Considering a complex system as a whole, an accurate analysis on its global dynamical behavior, with respect to the topology and global macroparameters has been carried out. Moreover, the effects of spatial diversity in complex lattices of nonlinear systems have been investigated. Spatial diversity actually helps systems in improving their pattern formation and self-organization aptitude. The fascinating conjecture that both irregularity in topologies and spatial dissymmetry generated by deterministic (although unpredictable) sources "naturally" improve cooperation and synchronization among the components of a complex system is still confirmed in this work by an accurate experimental analysis. This actually opens new perspectives in taming high-dimensional systems in which traditional feedback strategies are costly and time-consuming. The main result of this paper emphasizes the role that nonlocal connections, irregular topology and small spatial diversity in cell-based network play in pattern formation and self-organization. This is experimentally proved by showing that, by introducing these kinds of irregularity, synchronization and self-organization in Chua's circuit based networks occur with fewer cells than the case of "regular" networks.

\section{References}

Arena, P., Fortuna, L., Porto, D. \& Rizzo, A. [1999] "Selforganisation in arrays of nonlinear systems induced by chaotic perturbation: An experimental approach," Proc. IEEE-ISCAS99 Conf., Vol. V, Orlando, FL, pp. 479-482.

Braiman, Y., Lidner, J. F. \& Ditto, W. L. [1995] "Taming spatio-temporal chaos with disorder," Nature $\mathbf{3 7 8}$, November, 456-467.

Gell-Mann, M. [1994] The Quark and the Jaguar (Abacus, London).

Kaneko, K. [1990] "Clustering, coding, switching, hierarchical, ordering and control in a network of chaotic elements," Physica D41, 137-172.

Madan, R. N. (ed.) [1993] Chua's Circuit: A Paradigm for Chaos (World Scientific, Singapore).

Manganaro, G., Arena, P. \& Fortuna, L. [1999] Cellular Neural Networks: Chaos, Complexity and VLSI processing (Springer-Verlag).

Watts, D. J. \& Strogatz, S. H. [1998] "Collective dynamics of 'small-world' networks," Nature 393, June, 440-442. 